\title{
XEMIS2: A liquid xenon detector for small animal medical imaging
}

\author{
L. Gallego Manzano ${ }^{\mathrm{a}, *, 1}$, J.M. Abaline ${ }^{\mathrm{b}}$, S. Acounis ${ }^{\mathrm{a}}$, N. Beaupère ${ }^{\mathrm{a}}$, J.L. Beney ${ }^{\mathrm{a}}$, J. Bert ${ }^{\mathrm{h}}$, \\ S. Bouvier ${ }^{\mathrm{a}}$, P. Briend ${ }^{\mathrm{c}}$, J. Butterworth ${ }^{\mathrm{c}}$, T. Carlier ${ }^{\mathrm{d}}$, H. Chanal ${ }^{\mathrm{b}}$, M. Cherel ${ }^{\mathrm{e}}$, J.P. Cussonneau ${ }^{\mathrm{e}}$, \\ M. Dahoumane ${ }^{f}$, S. Diglio ${ }^{a}$, D. Giovagnoli ${ }^{\text {h}}$, J. Idier ${ }^{g}$, F. Kraeber-Bodere ${ }^{\mathrm{d}}$, F. Lefevre ${ }^{\mathrm{a}}$, \\ O. Lemaire ${ }^{a}$, P. Le Ray ${ }^{a}$, S. Manen ${ }^{b}$, J. Masbou ${ }^{a}$, H. Mathez ${ }^{\mathrm{f}}$, E. Morteau ${ }^{\mathrm{a}}$, N. Pillet ${ }^{\mathrm{b}}$, \\ L. Royer ${ }^{b}$, M. Staempflin ${ }^{c}$, J.S. Stutzmann ${ }^{a}$, R. Vandaele ${ }^{b}$, L. Virone $^{a}$, D. Visvikis ${ }^{\text {h }}$, Y. Xing ${ }^{\text {a }}$, \\ Y. Zhu ${ }^{\mathrm{a}}$, D. Thers ${ }^{\mathrm{a}}$ \\ a SUBATECH, IMT Atlantique, CNRS/IN2P3, Université de Nantes, 44307 Nantes, France \\ ${ }^{\mathrm{b}}$ LPC Clermont-Ferrand, 24 Avenue des Landais, Clermont-Ferrand, France \\ c AIR LIQUIDE Advanced Technologies Division, 2 rue Clémencière, F-38360 Sassenage, France \\ d Centre Hospitalier Universitaire de Nantes, 1 place Alexis-Ricordeau, 44093 Nantes, France \\ e INSERM U1232 équipe 13, 8 quai Moncousu, 44000 Nantes, France \\ ${ }^{\mathrm{f}}$ IPNL Université de Lyon, CNRS/IN2P3 UMR5822, France \\ ${ }^{g}$ LS2N, Ecole Centrale de Nantes, CNRS/In2p3, Université de Nantes, 44307 Nantes, France \\ ${ }^{\text {h }}$ UMR1101, LaTIM, CHRU Morvan, 2 avenue Foch, Brest, 29600, France
}

We report a new liquid xenon Compton camera, called XEMIS2 (XEnon Medical Imaging System), developed to image for the first time small animals using $3 \gamma$ imaging. This technique proposes an alternative to reduce the administered radiopharmaceutical activity while preserving diagnostic image quality using liquid xenon as detection medium. Given the success of its predecessor, a small dimension liquid xenon time projection chamber called XEMIS1, we are currently building a larger scale detector for preclinical applications. This second prototype, XEMIS2, is a single phase liquid xenon cylindrical camera with a large axial field of view for full-body small animal imaging. XEMIS2 holds around $200 \mathrm{~kg}$ of xenon and it has been designed to enable high-efficiency and simultaneous detection of the three $\gamma$-rays emitted by $\mathrm{a}^{44} \mathrm{Sc}$ radionuclide. The innovative geometry of XEMIS 2 will allow to discriminate and measure the position and deposited energy of most Compton interactions inside the detector. In order to manage such a large quantity of xenon, an innovative high-pressure subsystem known as ReStoX has been developed and successfully qualified. ReStoX allows to maintain the xenon in liquid state at the desired temperature and pressure, transfer the xenon into XEMIS2 and store it during long term periods. XEMIS2 will be installed at a non controlled radioactive area of Nantes Hospital. The goal is to evaluate image quality of a 20 min whole-body preclinical exam with an injected activity of $20 \mathrm{kBq}$.

\section{Introduction}

The reduction of the activity administered to patients, the decrease of the exposure time and the therapeutic follow-up of certain diseases, are three clear vectors that guide the future improvements especially for functional imaging techniques used in nuclear medicine. Reducing the administered activity will decrease patient radiation exposure, but at first sight it will also impact the image performances and lesion detectability by lowering image quality. To counteract these undesirable side effects, we propose an innovative low dose functional imaging technique, called $3 \gamma$ imaging, which is based on two new concepts: a liquid xenon (LXe) Compton camera and a new radiopharmaceutical labeled with a specific $3 \gamma$ emitter, the scandium- $44\left({ }^{44} \mathrm{Sc}\right)$ [1]. The intersection of the line-of-responses (LORs) from the coincidence detection of the two annihilation $\gamma$-rays and the Compton cone surface obtained from the third $\gamma$-rays allows the localization of the emission point with a resolution of about $1 \mathrm{~cm}$ (FWHM) along the LOR. Moreover, the reduction in the number of LORs will limit the number of disintegrations

\footnotetext{
* Corresponding author.

E-mail address: lucia.gallego.manzano@cern.ch (L. Gallego Manzano).

1 Now at CERN, CH-1211 Geneva 23, Switzerland.
} 
providing much faster images or good performance images at very low radiation doses. The principle of the $3 \gamma$ imaging technique is explained elsewhere [2].

A first prototype of a LXe Compton telescope, called XEMIS1 was successfully developed and tested. The detector is based on the principle of a liquid xenon time projection chamber (LXeTPC), which combines the measurement of both ionization and scintillation signals produced from the $\gamma$-ray interactions. The scintillation light $(178 \mathrm{~nm})$ is detected by an UV sensitive photomultiplier tube. The PMT is a $1^{\prime \prime}$ Hamamatsu R7600-06 PMT specifically developed to work at LXe temperature. The ionization charge carriers are accurately measured with a pixelated anode read-out by an ultra-low noise front-end electronics. The segmented anode is $2.5 \times 2.5 \mathrm{~cm}^{2}$ and it is divided in 64 pixels with a pitch of 3.1 $\mathrm{mm}$. The detector is also equipped with a Frisch grid used to shield the anode from the moving electrons in the drift region. To ensure a $100 \%$ electron transparency of the Frisch grid, an electric field in the gridanode gap of at least 5 to 10 times higher than the electric drift field is applied. The value of the field ratio depends on the characteristics of the grid [3]. For a more detailed description of the design of XEMIS1 and the different gap/Frisch grid configurations please refer to [4].

One of the advantage of a liquid xenon TPC (LXeTPC) is that it provides direct $3 \mathrm{D}$ information of each interaction inside the fiducial volume. Electron collection by the $3.1 \times 3.1 \mathrm{~mm}^{2}$ anode pixels gives 2D information (X-Y) with a resolution better than $1 \mathrm{~mm}$ [4]. The combination of both, scintillation and ionization signals, gives the absolute position of the interaction along the drift coordinate (Z-position) determined from the electron drift time, referred to the initial time of the event $t_{0}$, and the known electron drift velocity. Moreover, the deposited energy can be directly extracted from the amplitude of the measured charge signals.

The overall performances of XEMIS1 have been studied using a ${ }^{22} \mathrm{Na}$ source. The results and set-up description are published elsewhere [2,4]. The XEMIS1 prototype shows high Compton scattering efficiency, an energy resolution of 9\% (FWHM) for $511 \mathrm{keV}$ and a sub-millimeter spatial resolution. An angular resolution of $4^{\circ}$ was measured, which implies a resolution along the LOR smaller than $10 \mathrm{~mm}$ for a $5 \mathrm{~cm}$ distant source. This first prototype provided the experimental demonstration of the use of a LXe detector for $3 \gamma$ imaging. The reported results are compatible with the necessary requirements for small animal imaging and very promising for the future of the $3 \gamma$ imaging technique.

This paper is organized as follows. Section 2 details the characteristics of XEMIS2 and the readout electronics for the measurement of the ionization signal. The new cryogenic and purification systems are presented in Section 3. The paper concludes in Section 4 with the main aspects of the simulation performances of XEMIS2 and some preliminary results.

\section{XEMIS2: Small animal imaging with a liquid xenon Compton telescope}

\subsection{Detector description}

XEMIS2 is a single-phase LXeTPC designed for preclinical researches. Since the XEMIS project focuses on the development of low-dose wholebody imaging, the geometry of XEMIS2 has been designed to maximize the sensitivity of the detector by increasing the axial field-of-view (FOV). The detector consists of a cylindrical camera with $7 \mathrm{~cm}$ of inner radius, $19 \mathrm{~cm}$ of outer radius and a total active length of $24 \mathrm{~cm}$ filled with nearly $200 \mathrm{~kg}$ of xenon. It includes two identical back-to-back $12 \mathrm{~cm}$ long TPCs with a shared cathode. A cutout view of the design of XEMIS2 can be seen in Fig. 1.

Just like XEMIS1, the camera is adapted to detect both the ionization and scintillation signals produced after the interaction of an ionizing particle with the LXe [5]. Each TPC is equipped with a circular segmented anode to detect the electrons generated in the ionization process. The anodes are located at the edges of the TPCs and at $12 \mathrm{~cm}$ from

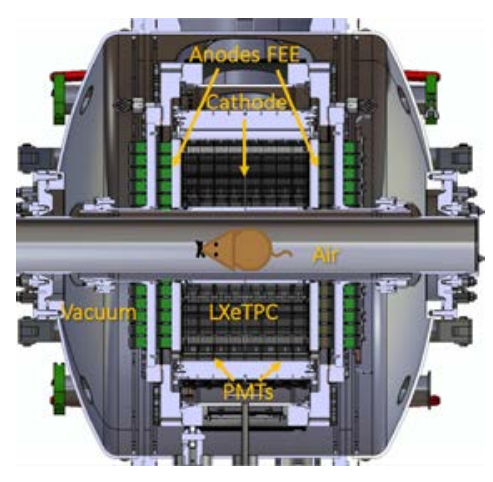

Fig. 1. Design of the XEMIS2 camera.

the common cathode. The total amount of ionization channels is 20480 with a pixel size of $3.1 \times 3.1 \mathrm{~mm}^{2}$. A mesh used as a Frisch grid is placed above each anode. Electrons are drifted towards the collecting electrodes by the influence of a homogeneous electric field provided by two sets of field shaping rings distributed along the $z$-axis.

The surface of the active area will be surrounded by $3801^{\prime \prime}$ PMTs to detect the VUV scintillation photons generated from the $\gamma$ ray interactions. The PMTs are completely immersed in the LXe. Since scintillation in LXe is a fast process with decay times of 2.2 and $27 \mathrm{~ns}$ (with a probability ratio of around $17 \%$ at zero electric field) [6], the detection of the scintillation light provides the initial time of the event $\mathrm{t}_{0}$. Moreover, it can provide a spatial pre-localization of the ionization signal reducing the dead time. In a preliminary phase, we use 64 PMTs distributed around the active volume of the detector. The purpose of this configuration is to show the possibility of performing a whole-body 20 min preclinical exam at very low injected activities down to $20 \mathrm{kBq}$.

As can be seen from Fig. 1, the detector is crossed side to side by a hollow tube made of Aluminum. The tube, which is placed outside the chamber, is in direct contact with the air and its purpose is to hold the small animal during the medical exam. A particular effort has been made to minimize the passive matter traversed by the $\gamma$-rays. The dimensions of the tube are $100 \mathrm{~mm}$ of diameter, a total length of $875 \mathrm{~mm}$ and 2.5 $\mathrm{mm}$ thick. To insulate the animal from the internal temperature of the cryostat and maintain it at room temperature while reducing the thermal losses, the tube is surrounded by a $7.5 \mathrm{~mm}$ thick vacuum insulation envelope. The inner container, that separates the LXe from the vacuum, has a thickness of $1.5 \mathrm{~mm}$ of stainless steel. A $3 \mathrm{~mm}$ LXe passive zone around the active volume is also required to immerse the electric field electrodes in order to ensure a uniform electric field.

The detector is built inside a vacuum-insulated double wall stainlesssteel vessel. The design of the cryostat was made to reduce the amount of LXe and the heat transfer into the detector. A frontal view of the XEMIS2 cryostat is presented in Fig. 2. The vacuum enclosure limits the convective heat transport, reducing the required cooling power necessary to maintain the xenon at the desired temperature. The xenon is reliably maintained at a pressure of 1.2 bar by means of a specific subsystem called ReStoX (Recovery and Storage of Xenon). A more detailed description of ReStoX is presented in the next section.

\subsection{Charge read-out electronics}

The ionization signals collected by the anodes are read-out by an ultra-low noise front-end electronics consisting of a 32-channels IDeF-X HD-LXe chip [7]. The 20480 pixels are connected to its own electronic channel providing independent analog signals. IDeF-X consists of a charge-sensitive preamplifier and a shaper, with a PZ filter for a tail cancellation stage. The signals are amplified and filtered with a peaking time of $1.39 \mu \mathrm{s}$. The ASICs are immersed in the LXe to minimize the thermal losses and to reduce the electronic noise ( $<100$ electrons). 


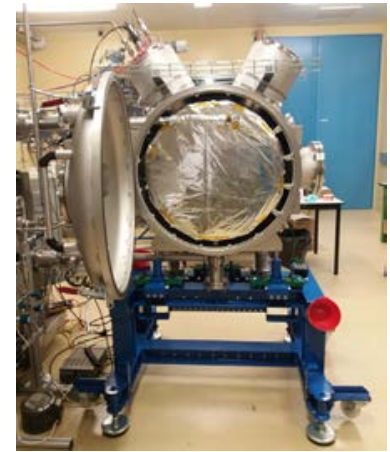

Fig. 2. Frontal view of the XEMIS2 cryostat. The inner chamber is wrapped with a Multilayer insulation (MLI) to reduce heat loss by thermal radiation.

To carry out an efficient and convenient data handling, a dedicated 32-channels low power circuit called XTRACT has been designed [4]. The analog pulses delivered by IDeF-X HD-LXe are then read-out by this new ASIC, in order to extract, from each individual pixel, the relevant information in terms of pulse height and arrival time of the signals. XTRACT includes three main features per channel: a self-trigger functionality with a very low threshold level (3 times the electronic noise) to maximize the detection efficiency, a Constant Fraction Discriminator (CFD) to measure the peak value and the signal arrival time, and an analog memory block that stores both amplitude and time informations of every triggered signal. The CFD parameters have been thoroughly studied and chosen to measure the pulse height at the zero crossing point of the bipolar CFD signal with a time resolution better than 250 ns for a signal-to-noise ratio of 3 [4]. The charge, time and pixel address informations from each channel delivered by XTRACT are read-out and digitized by a Process Unit board (PU). Both XTRACTs and the PU boards are located in the vacuum container at an estimated temperature of the order of $-80^{\circ}$ and $-30^{\circ}$ respectively.

Due to the low threshold level, the output data flow will be dominated by randomly generated noise triggered pulses. The expected data flow at a $3 \mathrm{MHz}$ reading rate is of the order of $10^{4}$ charge and time measurements per pixel per second. Data will be acquired and further reduced in FPGA boards by removing low amplitude signals isolated in space and time. In order to cover the entire anodes surface and read-out the 20480 pixels, XEMIS2 will be equipped with 640 IDeF-X LXe chips, 640 XTRACTs, 80 PU boards and 6 DAQ units.

\section{ReStoX: Recovery and storage of xenon}

The triple point of xenon is at $161.4 \mathrm{~K}$ which means that liquefaction at atmospheric pressure is moderately simple compared to other liquid noble gases [8]. However, dealing with a large amount of LXe requires an advanced technological concept to liquefy, store and transfer the xenon in an efficient and low consuming way. Moreover, since XEMIS2 has been conceived to be installed in a hospital center, the cryogenic infrastructure has to comply with the criteria of a medical imaging facility. For this reason, a sophisticated cryogenic storage and recovery system, called ReStoX, has been exclusively design for XEMIS2. Fig. 3 shows the XEMIS2 LXe cryogenic installation. The XEMIS2 facility can be divided in three different sub-systems: the XEMIS2 cryostat, the purification and recirculation systems and ReStoX.

ReStoX is a double-walled insulated stainless steel tank, that can hold up to 2801 of xenon. It has been design to maintain the xenon in liquid state at the desired temperature and pressure, distribute the xenon into the detector and also recover it in case of necessity. The inner vessel that holds the xenon, is $2 \mathrm{~cm}$ thick, $1 \mathrm{~m}$ height and it has a diameter of $610 \mathrm{~mm}$. To protect and insulate the inner vessel from convection heat transfer, it is enclosed in a vacuum insulation container. The vacuum jacket is $25 \mathrm{~cm}$ thick and it is filled with perlite insulation. Both stainless steel containers have a total weight of $440 \mathrm{~kg}$.

Since the scanner should only be operational during medical exams or calibrations tests, when the detector is not working, the chamber should be empty of LXe. This implies that a reliable and relative fast recovery and transfer system is necessary. The connection between XEMIS2 and ReStoX is therefore a key part of the design of the facility. When XEMIS2 is empty, the system needs to be completely isolated. On the other hand, when the camera is filled with LXe, the liquid level should be constant (pressure variations $<1$ mbar). Pressure regulation is performed by ReStoX through a massive aluminum condenser cooled down via a liquid nitrogen line. The aluminum block acts as a thermal buffer showing a wide cooling range from a few $\mathrm{W}$ up to $11 \mathrm{~kW}$ at $170 \mathrm{~K}$. Based on experimental results, without any cooling, the increase from LXe temperature to room temperature of the nearly $200 \mathrm{~kg}$ of xenon should be reached in about 1 year.

To remove any effect of the impurities that may affect the detection of both ionization and scintillation signals, the xenon is continuously purified through a re-circulation closed loop equipped with a gaseous purification system, based on an oil-free membrane pump and two rare-gas getters connected in parallel. A coaxial heat exchanger is used to transform the xenon from liquid to gaseous state with high efficiency. Attenuation lengths for electrons higher than $1 \mathrm{~m}(\sim 1 \mathrm{ppb}$ $\mathrm{O}_{2}$ equivalent) are expected after one week of circulation [4].

The whole cryogenic infrastructure has been successfully tested and qualified. $128 \mathrm{~kg}$ of liquid xenon have been transferred for the first time to XEMIS2. At a flow rate of about $3 \mathrm{~g} / \mathrm{s}$ the overall cooling and filling was achieved in nearly $20 \mathrm{~h}$. The recovery of the $128 \mathrm{~kg}$ of xenon was performed through a gravity assisted method allowing a complete and safety recovery in less than $10 \mathrm{~min}$. As a result, an outstanding and reproducible recuperation of the xenon from XEMIS2 to ReStoX at a flow rate of about 1 ton per hour is successfully achieved.

\section{The XEMIS2 simulated scanner and the $3 \gamma$ reconstruction algo- rithm}

To evaluate the performances of XEMIS2 and to develop a reconstruction method based on the $3 \gamma$ imaging principle, we conducted a GATE/GEANT4 Monte Carlo simulation [9]. Since this study focuses on the evaluation of the detector performances in terms of its acceptance, efficiency and event reconstruction capabilities, the simulation does not account for light production, electron drift and the electronic response of the detector. In a first study, we simulated a cylindrical water phantom ( $5 \mathrm{~cm}$ diameter and $12 \mathrm{~cm}$ length) uniformly filled with a total activity of $20 \mathrm{kBq}$ of ${ }^{44} \mathrm{Sc}$. To evaluate the image quality, a $1 \mathrm{~cm}$ diameter spherical point source with a contrast of 15 was added to the phantom at the center of the FOV. Among all simulated events, only those with at least four interaction points inside the camera (two from the two back-to-back $511 \mathrm{keV} \gamma$-rays and two from the third $1157 \mathrm{keV} \gamma$-ray) are considered for the reconstruction process. The algorithm is based on the direct 3D reconstruction, event-by-event, of the position of the emitter using the intersection of reconstructed LORs from the $511 \mathrm{keV}$ photons and the reconstructed Compton cone from the third $\gamma$-ray interactions. Only events with a single LOR-cone intersection point contribute to the image. Gamma-ray identification is possible using the deposited energy and the 3D spatial information per interaction point.

Results from the simulation showed a maximum sensitivity of $7 \%$ at the center of the FOV with an almost constant behavior throughout the entire phantom. Furthermore, we estimate from the simulation a precise localization of the emission point along the LOR of the order of 1 $\mathrm{cm}$ (FWHM). Image reconstruction is performed in a two-phase model. First, we obtain a raw image from the reconstructed points from the LOR-cone intersections. Then, in a second phase, a Maximum Likelihood Expectation Maximization (MLEM) iterative algorithm is applied to the raw image. For simplicity, data was modeled assuming a Poisson distribution with a constant Point Spread Function (PSF) along the entire 


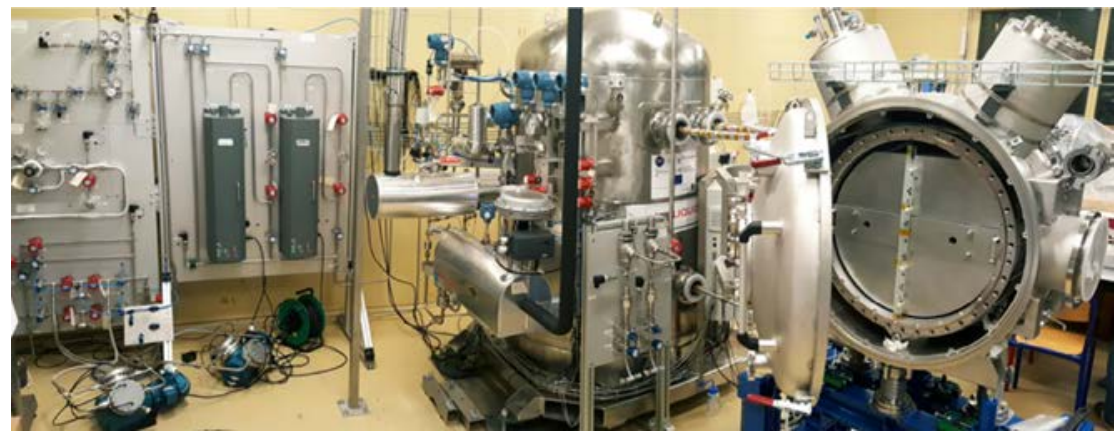

Fig. 3. Overview of the XEMIS2 cryogenics facility. From left to right: purification system, ReStoX and XEMIS2.
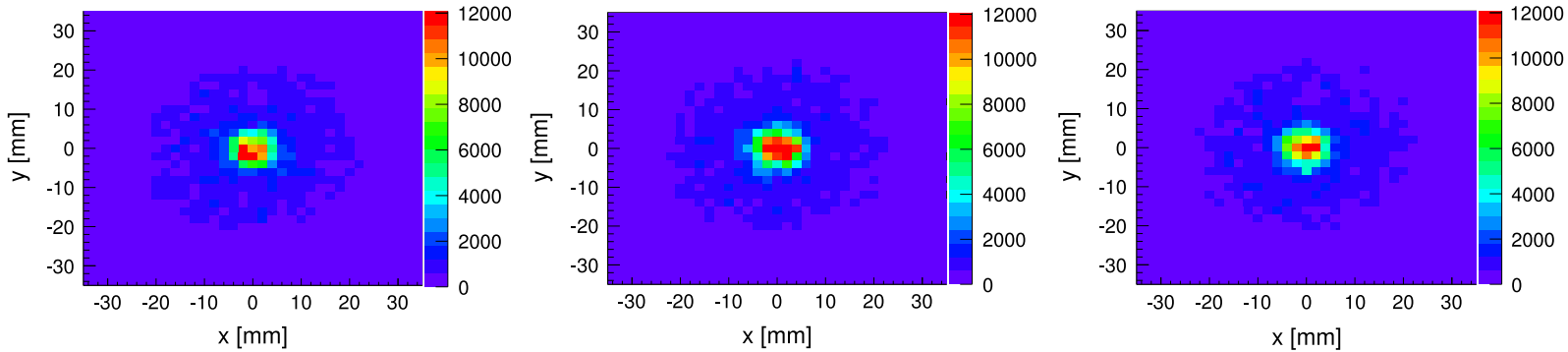

Fig. 4. Reconstructed image of a $20 \mathrm{~min}$ scan with $20 \mathrm{kBq}$ of injected activity for three different slices after 23 iterations of the MLEM algorithm. The voxel size is $(2 \times 2 \times 2)$ mm ${ }^{3}$.

FOV. Fig. 4 shows the reconstruction of the simulated phantom based on this new reconstruction algorithm. The results demonstrate that a $24 \mathrm{~cm}$ long LXe high sensitivity scanner, combined with the precise 3D localization offered by the $3 \gamma$ imaging reconstruction method, opens the possibility to obtain full-body good quality images at very low radiation doses and an acquisition time of $20 \mathrm{~min}$.

\section{Conclusion}

XEMIS is a new concept for functional imaging with high sensitivity and low dose performances. In this paper, we present a detailed description of the XEMIS2 camera: the first $3 \gamma$ whole-body small animal scanner. XEMIS2 is a monolithic liquid xenon detector with a $24 \mathrm{~cm}$ long axial field of view that holds nearly $200 \mathrm{~kg}$ of xenon. Based on the previous results obtained with XEMIS1 and those obtained from simulation, this innovative $3 \gamma$ LXe scanner will provide a high-sensitivity for small animal imaging compare to standard pre-clinical cameras [10]. The unique properties of XEMIS will open a new area for molecular imaging by reducing dramatically the injected activity and thus the effective dose, shortening scan times and allowing whole-body dynamic imaging paving the way to personalized medicine. XEMIS2 will be installed at the Nantes University Hospital for pre-clinical researches and it will serve as a preliminary step before considering a whole human body LXe scanner, XEMIS3.

\section{Acknowledgments}

The research presented in this paper has been funded by the E.U., the Region Pays de la Loire in France and by grants from the French National Agency for Research, "Investisement d'Avenir" ArronaxPlus Equipex ${ }^{o}$ ANR-11-EQPX-0004.

\section{References}

[1] S. Huclier-Markai, et al., Optimization of reaction conditions for the radiolabeling of dota and dota-peptide with $44 \mathrm{~m} / 44 \mathrm{sc}$ and experimental evidence of the feasibility of an in vivo pet generator, Nucl. Med. Biol. 41 (2014) 36-43.

[2] L. Gallego Manzano, et al., Xemis: A liquid xenon detector for medical imaging, Nucl. Instrum. Methods A 787 (2015) 89-93.

[3] O. Bunemann, et al., Design of grid ionization chambers, Can. J. Res. 27 A (1949) 191-206.

[4] L. Gallego Manzano, Optimization of a single-phase liquid xenon compton camera for $3 \gamma$ medical imaging, (Ph.D. thesis), Ecole des Mines de Nantes, July 2016

[5] E. Aprile, T. Doke, Liquid xenon detectors for particle physics and astrophysics, Rev. Modern Phys. 82 (2010) 2053-2097.

[6] T. Doke, Fundamental properties of liquid Argon, Krypton and Xenon as radiation detector media, Portugal. Phys. 12 (1981) 9-48.

[7] O. Lemaire, et al., Development of a readout electronic for the measurement of ionization in liquid xenon compton telescope containing micropatterns, IEEE NSS/MIC Conference, 2012, pp. 858-861.

[8] E. Aprile, A. Bolotnikov, A. Bolozdynya, T. Doke, Noble Gas Detectors, Wiley, 2006

[9] S. Jan, et al., Gate: A simulation toolkit for pet and spect, Phys. Med. Biol. 49 (2004) 4543-4561.

[10] M. Dahlbom, Physics of PET and SPECT Imaging, CRC Press, 2016. 